\title{
Journal of Traumatology and Clinical Orthopaedics
}

\section{Intrathoracic and pelvic migration of humeral fixation wires}

\author{
Millar DA ${ }^{1}$ and Langell $\mathrm{JT}^{1,2, *}$ \\ 1 Department of Surgery, University of Utah, Salt Lake City, Utah 84132, USA \\ ${ }^{2}$ Center for Medical Innovation, University of Utah, Salt Lake City, Utah 84132, USA
}

\begin{abstract}
The use of percutaneous fixation wires for orthopedic injuries may be associated with the complication of wire migration. Here, we report on the migration of three proximal humerus wires five weeks after insertion; one of which we believe may be the longest distance traveled for a percutaneous wire into the pelvis. The patient is a 67-year-old female who underwent percutaneous pinning with four Kirshner wires for a left proximal humerus fracture. At a five-week follow up appointment she underwent removal of one of these wires secondary to ongoing pain. Radiographs showed the other three wires were no longer at the site of repair. Subsequent spiral CT scans of the chest, abdomen and pelvis showed migration of the remaining three wires into the mediastinum, pelvis, and left posterorlateral back. The intrathoracic wire traversed the subclavicular tissue, anterior to the left common carotid artery and terminated anterior to the trachea, abutting the brachiocephalic artery. The pelvic wire traversed the preperitoneal space to abut the left external iliac vein and sigmoid colon. All wires were successfully removed surgically through small incisions with the aid of ultrasound and fluoroscopy and without complication. The patient made an uneventful recovery. Proposed theories for wire migration include bone resorption, extremity motion, muscular contraction, respiratory motion and negative intrathoracic pressure. Regardless of cause, caution must be taken to avoid wire migration and vigilant follow up with radiographs can help reduce morbidity associated with this complication.
\end{abstract}

Keywords: surgery; Kirshner wire; humeral fixation; k-wire; wire migration

\section{Introduction}

Fractures of the proximal humerus are common orthopedic injuries. The use of percutaneous pins and wires for fixation of humeral fractures are also common, but can be associated with complications, including wire migration. Previous reports of fixation wire migration have previously been documented in the surgical literature. There are reports of wire migration from the pelvis and humerus into the mediastinum, trachea, bladder, lung, great vessels, heart, and spleen [1-9]. Here, we report on the migration of three proximal humerus fixation wires just five weeks after insertion, one of which we believe may be the longest distance traveled for a percutaneous wire into the pelvis.

\section{Case report}

A 67-year-old morbidly obese female with severe muscle weakness, secondary to multiple sclerosis, suffered a left proximal humeral fracture secondary to a fall in her nursing home. She underwent percutaneous pinning with placement of four threaded Kirshner wires (K-wire). At a five week follow up appointment she underwent removal of one of these wires, the remaining three could not be located on physical examination or an upper extremity radiograph conducted by the treating orthopedic surgeon. The patient was subsequently transferred to our emergency department where spiral CT scans of the chest abdomen and pelvis showed migration of the additional three wires.
One K-wire was found medial to the left humeral head traversing the subclavicular soft tissue abutting the left internal jugular vein, traversing anterior to the left common carotid artery and terminating anterior to the trachea abutting the brachiocephalic artery. A second K-wire was found in the soft tissue of the left posterior chest wall with the tip in the latissimus dorsi muscle. The third was located in the pelvis abutting the left external iliac vein and sigmoid colon while extending anteromedially to the rectus abdominis (Figures 1-3). A CT scan with gastrografin enema was subsequently performed demonstrating no evidence of colonic perforation. The patient was afebrile, hemodynamically stable and otherwise asymptomatic with the exception of ongoing left shoulder pain at the time of presentation.

*Corresponding author: John Langell, Center for Medical Innovation, University of Utah, 10 North 1900 East Eccles Library, Room 15, Salt Lake City, Utah 84132, USA. Tel.: +1-801-587-7288; Fax: +1-801-587-9370; Email: john.langell@hsc.utah.edu

Received 18 April 2016 Revised 7 June 2016 Accepted 21 June 2016 Published 30 June 2016

Citation: Millar DA, Langell JT. Intrathoracic and pelvic migration of humeral fixation wires. J Traumatol Clin Orthop. 2016; 1(1):1-3. DOI: 10.14312/23998180.2016-1

Copyright: (c) 2016 Millar DA, et al. Published by NobleResearch Publishers. This is an open-access article distributed under the terms of the Creative Commons Attribution License, which permits unrestricted use, distribution and reproduction in any medium, provided the original author and source are credited. 


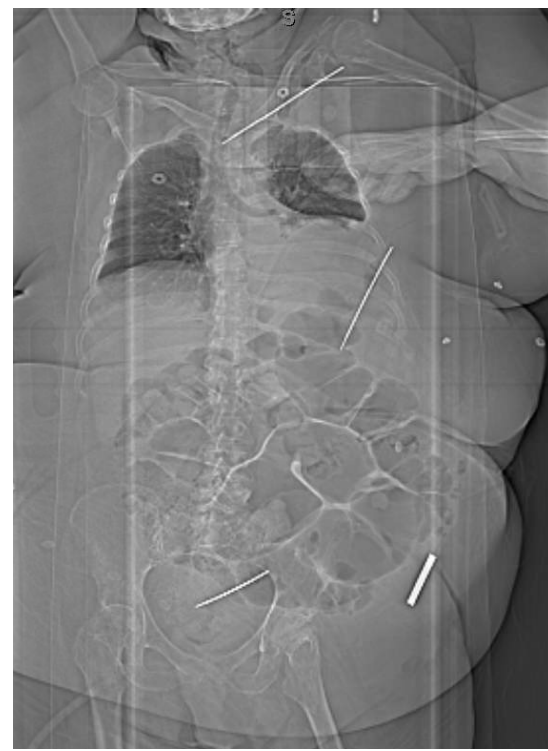

Figure 1 Scout CT scan demonstrating a K-wire in the mediastinum, a K-wire in the thorax and a K-wire in the pelvis.

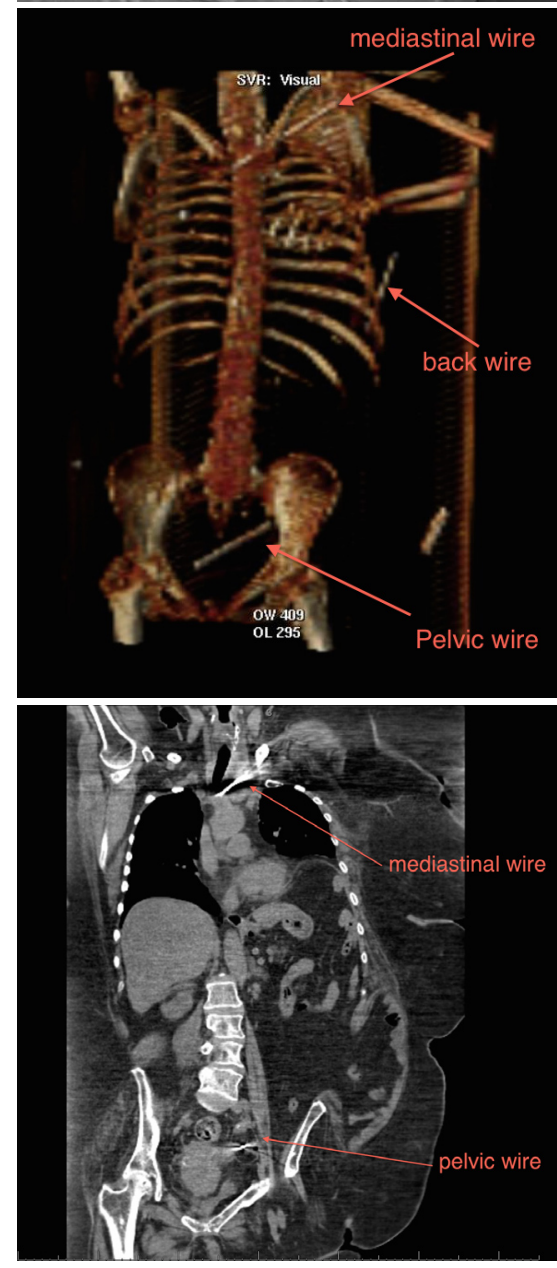

Figure 2 A biplanar CT scan reconstruction demonstrating K-wire location relative to bony anatomical landmarks.

Figure 3 A coronal plane CT scan image demonstrating the mediastinal and pelvic K-wire locations relative to vital anatomic structures.

The patient was taken to the operating room for removal of the migrated hardware. While awake and sitting upright the wire in her left posterior back was located using ultrasound and fluoroscopy. After injecting local anesthetic we made a $3 \mathrm{~cm}$ incision over the wire and successfully removed it without complication. The patient was then placed supine under general anesthesia with the cardiothoracic surgery team available on standby. Fluoroscopy was again used to locate the migrated wire in the left chest. A $5 \mathrm{~cm}$ incision was made in the deltopectoral groove and the dissection was carried down through the pectoralis major using a muscle splitting technique. We mobilized the pectoralis minor along the superior border and encountered the wire near the chest wall. It was then backed out along its track and removed using a separate skin incision in the axilla without complication.

The wire in the pelvis was then located using fluoroscopy. A $5 \mathrm{~cm}$ incision was made over the region of the wire; we opened the preperitoneal space and dissected to the level of the fascia. Once visualized, the wire was extracted without difficulty. It appeared that the tip of the K-wire was likely resting on the iliac wing. The wire was removed and no evidence of enteric contamination or bleeding was noted. All extracted wires were found to be threaded at the proximal end and intact without any introduced bends, kinks or deformations to the structure of the wire.

Postoperative imaging included chest radiography and an esophagram. There was no evidence of pneumothorax, pneuomediatstinum, or esophageal perforation. The patient had an uneventful recovery and was discharged from the hospital back to her skilled nursing facility on postoperative day four.

\section{Discussion}

While migration of orthopedic fixation hardware is rare, it is a known complication and requires vigilant follow up. The cause for wire migration is debatable. Proposed theories include bone resorption, gravitational forces, upper extremity motion, muscular contraction, respiratory motion and negative intrathoracic pressure [9].

Multiple case reports have emerged documenting the migration of fixation wires primarily from the shoulder girdle the pelvis. Both locations have yielded wires migrating predominately into adjacent body cavities and into vital organs [1-9]. There have also been reports of pins traveling long distances against gravity from the pelvis into the mediastinum [8]. In addition, others have reported cases of aortic laceration and pericardial tamponade from pins placed into the shoulder girdle [2]. Having reviewed the current literature, we believe the above described wire migration into the pelvis demonstrates longest reported distance traveled for a fixation pin. While this case did not result in death or organ dysfunction it reinforces the need for aggressive monitoring of fixation wire location via standard radiography during the course of treatment as well as taking precautionary measures such as bending the distal end of the wire to inhibit migration.

\section{Conclusion}

Fixation of orthopedic injuries using fixation pins and wires is a common and acceptable practice that usually produces excellent results with low risk. Wire migration is a known complication and unexpected morbidity can be avoided by ensuring proper securing of the fixation devices and regular follow up. Fixation hardware should be removed as soon as reasonably possible.

\section{Conflicts of interest}

The authors declare no conflicts of interest. 


\section{References}

[1] Gulcan O, Sezgin AT, Bolat B, Turkoz R. Right ventricular penetration and cardiac tamponade as a late complication of Kirschner wire placement in the sternoclavicular joint. Interact Cardiovasc Thorac Surg. 2005; 4(4):295-296.

[2] Lin F, Pu Q, Ma L, Liu L. Pericardial tamponade caused by a migratory Kirschner wire. Eur J Cardiothorac Surg. 2016; 49(3):1012.

[3] Potter FA, Fiorini AJ, Knox J, Rajesh PB. The migration of a Kirschner wire from shoulder to spleen: brief report. J Bone Joint Surg Br. 1988; 70(2):326-327.

[4] Nordback I, Markkula H. Migration of Kirschner pin from clavicle into ascending aorta. Acta Chir Scand. 1985; 151(2):177-179.

[5] Cameliere L, Rosat P, Heyndrickx M, Le Rochais J, Icard P. Migration of a Kirschner pin from the shoulder to the lung, requiring surgery. Asian Cardiovasc Thorac Ann. 2013; 21(2):222-223.

[6] Nakayama M, Gika M, Fukuda H, Yamahata T, Aoki K, et al. Migration of a Kirschner wire from the clavicle into the intrathoracic trachea. Ann Thorac Surg. 2009; 88(2):653-654.

[7] Wu YH, Lai CH, Luo CY, Tseng YL. Tracheoinnominate artery fistula caused by migration of a Kirschner wire. Eur J Cardiothorac Surg. 2009; 36(1):214-216.

[8] Ballas R, Bonnel F. Endopelvic migration of a sternoclavicular K-wire. Case report and review of literature. Orthop Traumatol Surg Res. 2012; 98(1):118-121.

[9] Kumar S, Singh SK, Jayant SK, Agarwal S, Parmar KM, et al. Forgotten Kirschner Wire Causing Severe Hematuria. Case Rep Urol. 2014; 2014:305868. 